\title{
Chiral Seismic Attenuation with Acoustic Metamaterials
}

\author{
Hector Torres-Silva ${ }^{1}$, Diego Torres Cabezas ${ }^{2}$ \\ ${ }^{1}$ Escuela de Ingeniería Eléctrica Electrónica, Universidad de Tarapacá, Arica, Chile; ${ }^{2}$ Modernización y Gobierno Electrónico, Mini- \\ sterio Secretaría General de la Presidencia, Santiago, Chile. \\ Email: htorres@uta.cl
}

Received November $4^{\text {th }}, 2012$; revised December $6^{\text {th }}, 2012$; accepted December $18^{\text {th }}, 2012$

\begin{abstract}
We study the analogy between the linear elasticity theory equations and classical Maxwell equation with chiral effects and we propose a new method of an earthquake-resistant design to support conventional aseismic designs using acoustic metamaterials. We suggest a simple and practical method to reduce the amplitude of a seismic wave exponentially. Our device is like an attenuator of a chiral seismic wave. Constructing a cylindrical shell-type waveguide that creates a stop-band for the chiral seismic wave, we can convert the wave into an evanescent wave for some frequency range without touching the building we want to protect.
\end{abstract}

Keywords: Chiral Metamaterial; Seismic Wave; Acoustic Properties

\section{Introduction}

Earthquakes are the result of sudden release of huge amount of energy in the Earth's crust that produces seismic waves. There is a lot of observational evidence concerning rotational motions on the Earth's surface excited by earthquakes, we can infer the existence of rotational motions in earthquake focal zones. It is observed that rotational motions (twist) in earthquake sources naturally excite rotational seismic waves, solitons and chiral waves [1-3].

The key idea of this article consists in the description of rotational seismic waves into a chiral regime $[4,5]$ by considering chiral rotational seismic waves excited in earthquake sources in strong earthquakes. We can distinguish two kinds of rotational seismic waves: 1) rotational longitudinal waves, that is, PR waves; and 2) rotational shear waves, that is, SR waves. Rotational seismic waves propagate faster in solid rocks and much slower in fractured media along tectonic faults. It has been observed that these waves may have a form of rotational seismic solitons and that they can trigger earthquakes [6]. Because of the fact that solitons can propagate without any loss of energy, these waves are extremely important carriers of seismic energy.

It is the collapse of structures that causes extensive damage and loss of life during earthquakes. Aseismic capabilities are highly relevant to public safety and a large amount of research has gone into establishing practical analysis and design methods for them.

The main principles underpinning the protection of structures from seismic waves are applied to waves of force that travel through the Earth, which is an elastic body. Travelling pressure waves are always generated with longitudinal polarization. This means that the ground is alternately compressed and dilated in the direction of propagation. Travelling shear waves are transverse, or perpendicular to the direction of propagation. One of the effects of the metamaterial is to de-couple these components of the seismic wave. By applying these principles, it is possible to design a cylindrical, seismic metatmaterial cloak to remedy the destructive effects of coupled pressure and shear waves that would travel through the same earthen geometrical plane as the structure. The components of the seismic waves should work together to disrupt the elastic earth, on which a building is anchored.

Recent development of metamaterial science opens a new direction to control the seismic waves. Farhat et al. proposed a design of a cloak to control bending waves propagated in isotropic heterogeneous thin plates [7-9].

Their cloaking of shear elastic waves pass smoothly into the material rather than reflecting or scattering at the material's surface. In this paper we propose a method to control seismic waves by using a new class of materials called metamaterials. Metamaterials are artificially engineered materials which have a special property of negative refractive index.

We present here a solution that a metamaterial acts as an attenuator by converting the destructive seismic wave into an evanescent wave by making use of the imaginary velocity of stop-band of the wave.

There are many representations to express the scale of earthquakes. Among them the magnitude that comes 
from the amplitude of the seismic waves is most important. The common form of the magnitude, $R$, is the Richter-scale defined by comparing the two amplitudes in a logarithmic scale as $R=\log E / E_{0}$, where $E$ is the maximum amplitude of the seismic wave and $E_{0}$ is the maximum amplitude of the background vibration and order of $\mu \mathrm{m}$. We focus here on how to reduce the amplitude of the seismic wave by using the properties of chiral metamaterials. In Section 2 of this paper, we develop a theory for chiral seismic waves. Based on the similarity of elastodynamics and electrodynamics we discuss the region of elastic metamaterial with negative modulus and negative density that enable a seismic wave becomes evanescent wave. In Section 3, we discuss a scheme of seismic attenuator. The novelty of this proposal is that the attenuation is due to the combined effect of metamaterial and evanescence in $\mathrm{S}$ waves.

\section{Chiral Theory: Negative Modulus, Negative Density}

In connection with seismic events, the Elastodynamics is linked with Electrodynamics through the transverse waves. Seismic waves encountering interfaces that separate rocks of different elastic properties also undergo reflection, refraction, and scattering phenomena and chiral materials are noncentrosymmetric due to handedness in their microstructures. The elastic field behavior in a chiral medium is readily described herein using the governing equations and constitutive relations for noncentrosymmetric, isotropic micropolar materials. Accordingly, linearly polarized longitudinal waves and left and right circularly polarized transverse waves are eigenstates for elastic waves in the chiral medium. There are two classes of seismic body waves which travel through the interior of the Earth: $\mathrm{P}$ waves and $\mathrm{S}$ waves. $\mathrm{P}$ waves are the fastest seismic waves, and consequently, the first to arrive at any given location. Torsion waves, often called $\mathrm{S}$ waves, represent the spiraling motion of particles twisting between inner structures. $\mathrm{P}$ waves are usually the first to be recorded on a seismogram because they travel the fastest. S waves usually have more height, or amplitude, than $\mathrm{P}$ waves.

We will be started with consideration of an analogy between the linear elasticity theory equations and classical Maxwell equation with chiral effects. Chiral media have the property to split two degenerate modes [10-14]. For a chiral medium with an electric or magnetic resonance, a negative refractive band is created and there is a continuous transition between the negative and positive bands, as described in [10]. Generally, a structure is said to be chiral if it is not identical to its mirror image, and chirality has as a result the macroscopic rotation of the wave polarization. Let us say that it is the elastic continuum that supports propagation of both longitudinal and transverse waves. The basic equation of the linear elasticity theory is the Lame equation, which takes the following form

$$
-\ddot{u}+c_{L}^{2} \nabla \operatorname{div} \boldsymbol{u}-c_{T}^{2} \nabla \times(\nabla \times \boldsymbol{u})=0
$$

where the vector $\boldsymbol{u}$ represents the vector of displacements is a elastic medium, $c_{L, T}$ are the velocities of propagation of the longitudinal ( $\mathrm{P}$ waves) and transverse (S waves) waves correspondingly. In order to separate the wave processes explicitly we introduce the chiral seismic parameter $\zeta$ which take into account the mode conversion from $\mathrm{P}$ to $\mathrm{S}$ waves with the time varation as

$$
\frac{\partial}{\partial t} \rightarrow \frac{\partial}{\partial t}(1+\zeta \nabla \times)
$$

Here we identify $\boldsymbol{E}_{S}=-\boldsymbol{u}, \boldsymbol{H}_{S}=-c_{T} \nabla \times \boldsymbol{u}, W=c_{L} \nabla \cdot \boldsymbol{u}$, so using the Born-Fedorov formalism

$$
\boldsymbol{E}_{T S}=\varepsilon \boldsymbol{E}_{S}+\varepsilon \zeta\left(\nabla \times \boldsymbol{E}_{S}\right)
$$

and

$$
\boldsymbol{H}_{T S}=\mu \boldsymbol{H}_{S}+\mu \zeta\left(\nabla \times \boldsymbol{H}_{S}\right),
$$

the equivalent Maxwell equations are

$$
\begin{gathered}
\nabla \times \boldsymbol{E}_{T S}=-\frac{1}{c_{T}}\left(\mu \boldsymbol{H}_{S}+\mu \zeta \nabla \times \boldsymbol{H}_{S}\right) \\
\nabla \times \boldsymbol{H}_{T S}=\frac{1}{c_{T}}\left(\varepsilon \boldsymbol{E}_{S}+\varepsilon \zeta \nabla \times \boldsymbol{E}_{S}\right)
\end{gathered}
$$

Solving these equations we have that the effective phase velocity of S-waves is $v_{S}=c_{T}\left(1 \pm \zeta k_{0}\right)$ where $k_{0}$ is the wavevector without $\zeta$ and the wave equation is

$$
\nabla_{s}^{2} \boldsymbol{E}_{T S}+\frac{\omega^{2}}{v_{S}^{2}} \boldsymbol{E}_{T S}=-\nabla_{s}^{2} \boldsymbol{u}-\frac{\omega^{2}}{v_{S}^{2}} \boldsymbol{u}=0
$$

Metamaterials with negative electromagnetic constitutive parameters give new propagation characteristics for electromagnetic waves [10-14]. Negative permittivity occurs naturally due to the plasma oscillation, but negative permeability became available only artificially [10]. A composite structure consisting of the elements of negative permittivity (metal wire) and negative permeability (split ring resonator) resulted in simultaneously negative values of $\varepsilon$ and $\mu$.

Although from an electromagnetic point of view, chiral homogeneous material can be described by different specific equations, by analogy between elastodynamics and electrodynamics in this work we use the BornFedorov equations as the most suitable for applications of our interest [5-6]. Here the density of matter is equivalent to the electric permittivity $\rho \leftrightarrow \varepsilon$, and the Lamé parameter $\mu_{S}$ is equivalent to the reciprocal of the magnetic permeability $\mu_{S} \leftrightarrow 1 / \mu$. The speed of 
transversal seismic S-waves without chirality is given by $c_{T}=1 / \sqrt{\rho / \mu_{S}}$. The chiral effect given by the factor $\left(1 \pm \zeta k_{0}\right)$ which modify the phase velocity $c_{T}\left(k_{0}=\omega / c_{T}\right)$.

On the other hand, acoustic waves are created by compressibility or elasticity of the medium. Young's modulus, $Y$, is a one-dimensional compressibility defined by $\Delta p=Y \Delta l / l$, where $\Delta p$ is the pressure or stress and $l$ is the length. Shear modulus, $B_{S}$, is a two-dimensional one for a surface wave defined by $\Delta p=B_{S} \Delta x / h$, where $\Delta x$ is the horizontal shift and $h$ is the height of the object. Bulk modulus, $B_{V}$, is a three-dimensional one for a body wave defined by $\Delta p=B_{V} \Delta V / V$.

Seismic medium of Earth crust can be considered as an accumulation of infinite number of elastic plates. Although the seismic surface wave is not pure two dimensional, the velocity is mainly dependent upon the density, $\rho_{S}$ and shear modulus, $B_{S}$, of the seismic medium. Seismic wave is a kind of acoustic wave and every acoustic wave propagates following two wave equations in principle. Assuming the plane wave time dependence $\exp (i \omega t)$, the pressure, $p$, and the velocity, $\boldsymbol{v}_{S}$ of the S-wave in the two dimensions are expressed as the Newton's law [15].

$$
\nabla_{s} p=i \omega \rho v_{S}
$$

and the continuity equation

$$
i \omega p=B_{S} \nabla \cdot v_{S}
$$

where $\nabla$ is the Laplacian operator at the surface, $p$ is the pressure, $\omega$ is the angular frequency of the wave, and $\boldsymbol{v}_{S}$ is the velocity.

The Equations (4) and (5) generates the wave equation as

$$
\nabla_{s}^{2} p+\frac{\omega^{2}}{\boldsymbol{v}_{S}^{2}} p=0
$$

if we compare Equation (3) with Equation (6), we complete the analogy and connection between the chiral electromagnetism and the acoustic seismic waves. The velocity of the seismic wave is

$$
v_{S}=\sqrt{B_{S} / \rho}\left(1 \pm \zeta k_{S}\right)
$$

The Equation (4) is the counterpart of the Ampere's law and Equation (5) is of the Faraday's law by the analogy of electromagnetism and acoustic mechanics.

If the shear modulus $B_{S}$ or the density $\rho$ becomes negative, the velocity becomes imaginary. Then, so does the refractive index, $\mathrm{n}$, or the inverse of the velocity as $n=v_{0} / v_{S}=v_{0} \sqrt{\rho / B_{S}}$, where $v_{0}$ is the background velocity. Since $k_{S}=2 \pi n / \lambda_{S}$ the wavevector becomes imaginary, too. Therefore, the imaginary wavevector makes the amplitude of the seismic wave become an evanescent wave. We call it an attenuator. Negative shear modulus of elastic media has been studied and realized very recently [16-18] (Helmholtz resonators with the negative bulk modulus). The resonance of accumulated waves in the Helmholtz resonator reacts against the applied pressure at some specific frequency ranges. Then, the negative modulus is realized by passing the acoustic wave through an array of Helmholtz resonators.

In Figure 1, we illustrate the fact that if the normalized frequency is between 1.3 and 1.4, absorption of acoustic energy is maximum and the real part of $\mathrm{B}$ is negative. If simultaneously the real part is negative density frequency for that segment, then it would have an effect metamaterial which would make the phase velocity of the wave would be opposite to S-wave vector and enhance the global attenuation. (see Equations (9) and (11) below).

Therefore, the sound intensity decays exponentially at some resonant frequency ranges. In general the elastic material is described by three independent effective parameters of $B_{S}, B_{V}$, and $\rho$ [2]. Acoustic waves from the modulus share fundamental properties of sound waves.

From the formalism of electromagnetic response in metamaterials, effective electric permittivity and effecttive magnetic permeability show negative values at some specific frequency ranges around resonances $[19,20]$. The Helmholtz resonator is a realization of an electrical resonance circuit by mechanical correspondence. It is known as that the ferriplasmon frequency in metals or in an array of ring metal wires produces the magnetic permeability as [16]

$$
\mu=\mu_{0}\left[1-\frac{\omega_{o m}^{2}}{\omega(\omega+i \Gamma)}\right]
$$

where $\omega_{o m}$ is the ferriplasma frequency and $\Gamma$ is a

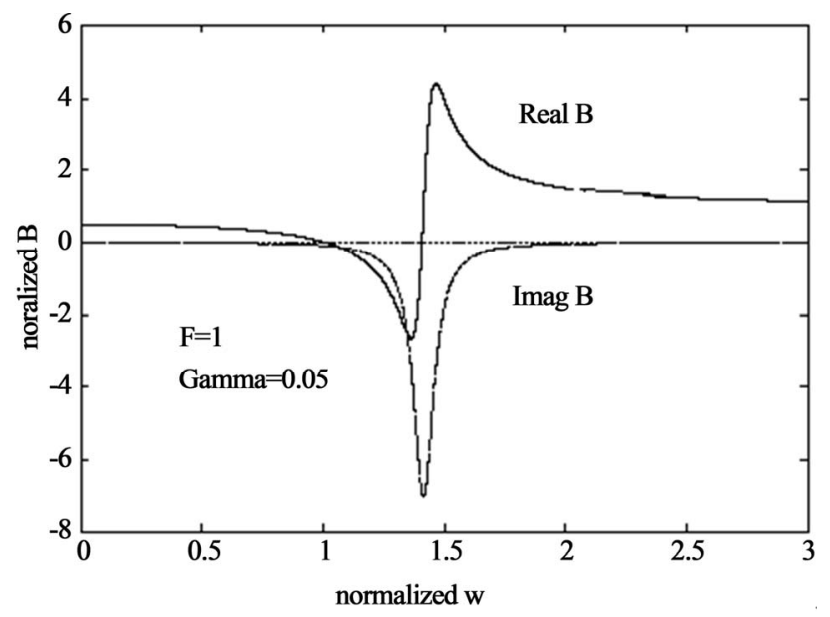

Figure 1. Illustration of real and imaginary parts of the normalized effective shear modulus. The negative peak of the imaginary part means an energy absorption. 
loss by damping.

Considering the structural loss, the general form of the effective shear modulus, $B_{e f f}$, is given similarly with the general form of the bulk modulus as [10-14]

$$
\frac{1}{B_{e f f}}=\frac{1}{B_{S}}\left(1-\frac{F_{m} \omega_{o m}^{2}}{\omega^{2}-\omega_{o m}^{2}+i \Gamma \omega}\right)
$$

where $\omega_{0}$ is the resonance frequency and $F$ is a geometric factor $[15,21,22]$. The real and imaginary part of the $B_{\text {eff }}$ is plotted in Figure 1 at specific values of $F$ and $\Gamma$. The real part can be negative at resonance and slightly increased frequency ranges. The negative range of the real part is the stop-band of the wave. When the imaginary part, the loss, is small compared with real part, the effective shear modulus has negative value at $1<\omega / \omega_{0 m}<\sqrt{1+F_{m}}$. Similar considerations can be made to dielectric plasma.

$$
\varepsilon=\varepsilon_{0}\left[1-\frac{\omega_{p}^{2}}{\omega(\omega+i \Gamma)}\right]
$$

which will be proportional to density $\rho$, so

$$
\rho_{e f f}=\rho_{S}\left(1-\frac{F_{e} \omega_{o p}^{2}}{\omega^{2}-\omega_{o p}^{2}+i \Gamma \omega}\right)
$$

which may be negative too and in this case the phase velocity is negative near of resonance conditions ( $B_{\text {eff }}$ and $\rho_{\text {eff }}$ are simultaneously negatives) or when $\left(1 \pm \zeta k_{S}\right)<0$.

\section{Seismic Attenuator}

We can build an attenuator or a barrier of a seismic wave by filling-up many resonators under the ground around the building that we want to protect. Then, the amplitude of the seismic wave that passed the waveguide is reduced exponentially by the imaginary wavevector at the frequency ranges of negative modulus. Mixing up many different kinds of resonators will cover many different corresponding frequency ranges of the seismic waves. The chiral factor can enhance this attenuation if we take $\left(1 \pm \zeta k_{0}\right)$ with the appropriate sign [23-26].

If we assume that the plain seismic wave of wavelength $\lambda$ propagates in $x$-direction, the amplitude of the wave reduces exponentially as

$$
E \exp \left(i k_{S} x\right)=E \exp \left(-2 \pi|n| x /\left(1 \pm \zeta k_{0}\right) \lambda\right)
$$

Let the initial seismic wave, that is, before entering the waveguide, have amplitude $E_{i}$ and magnitude $R_{i}$, and final seismic wave, that is after leaving the waveguide, have amplitude $E_{f}$ and magnitude $R_{f}$. Then, $E_{f}$ is written as

$$
E_{i} \exp \left(-2 \pi|n| x /\left(1 \pm \zeta k_{0}\right) \lambda\right)=E_{f}
$$

The amplitude of the seismic wave reduces exponenttially as passing the waveguide of metamaterials. We can rewrite Equation (9) with the definition of the magnitude in

$$
R=\log \frac{E}{E_{0}} \text { as } E_{0} 10^{R_{i}} \exp \left(-2 \pi|n| x /\left(1 \pm \zeta k_{0}\right) \lambda\right)=E_{0} 10^{R_{f}}
$$

Taking logarithms both sides of Equation (10), we obtain the width of the waveguide, $x \rightarrow \Delta X$, as

$$
\Delta X=\frac{\ln 10}{2 \pi} \frac{\lambda \Delta R\left(1 \pm \zeta k_{0}\right)}{|n|}=0.366 \frac{\lambda \Delta R}{|n|}\left(1 \pm \zeta k_{0}\right)
$$

where

$$
\Delta R=R_{i}-R_{f} k_{0}=\omega / c_{T}, k_{S}=\omega /\left(c_{T}\left(1 \pm \zeta k_{0}\right)\right)
$$

For example, if the refractive index is $n=2$ and $\zeta k_{0}=0$, the wavelength of the surface wave is $\lambda=100 \mathrm{~m}$, we need $\Delta X=16.80 \mathrm{~m}$ to reduce $\Delta R=1$. If $\zeta k_{0}=0.5$ we need $\Delta X=8.40 \mathrm{~m}$. That is the chiral factor can substantially reduce the volume of materials, with the significant reduction in engineering costs.

If the aseismic level of the building is $R=6$ and the width of the waveguide surrounding the building is about $\Delta x=50 \mathrm{~m}$, then the effective aseismic level of the building is increased to $R=8.73$. Therefore, a high refracttive index material can be obtained by the factor $\left(1 \pm \zeta k_{0}\right)$ for a narrow attenuator $(\Delta X)$. In civil engineering earthquake proofing methods must be practical, that is, with great versatility, simplicity and easy to construct.

The resonator should be easy to build. We show an example of a resonator in Figure 2.

The size of the cylinder can be estimated from the analogy between electric circuits and mechanical pipes.

An example of chiral metamaterials, is chiral Swiss Rolls, which are constructed by wrapping a conducting sheet around a cylindrical mandrel in a helical shape (as shown in Figure 2). The chiral design ca exhibit enormous

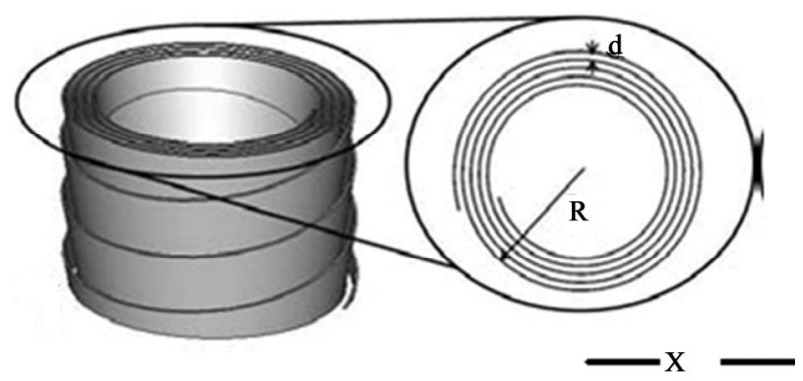

Figure 2. A chiral Swiss roll, where $d$ is the gap between the conducting sheets and $\mathrm{R}$ the outer radius $X=n d$. A sample of a meta-cylinder can have 4 side holes or more in the cylinder mantle. The size of the cylinder is less than the wavelength of the surface waves. 
chirality $\zeta$, at least 50 times larger than other chiral structures previously reported in the literature. This property ensures the change of a wave polarization by $90^{\circ}$ in less than a wavelength. Hence, chiral Swiss Rolls support a negative refractive band for one-wave polarization, which is predicted analytically and calculated numerically and therefore a macroscopic resonant magnetic behaviour is created. Chirality arises from the helical shape of the structure.

An example of the design of the meta-cylinder for the seismic frequency range is followings: the diameter of the hole is order of $0.1 \mathrm{~m}$, the thickness of the cylinder is order of $0.1 \mathrm{~m}$, and the volume inside is order of $\mathrm{m}^{3}$. Since the meta-cylinder is considerably smaller than the corresponding wavelengths, the array meta-cylinders behave as a homogenized medium. The chiral $\zeta$ factor can reduce the phase velocity so the S-wave can be stopped.

The shape of the meta-cylinder is neither necessary to be circular nor to have 4 holes. It could be any form of a concrete box with several side holes. Cubic or hexagonal boxes would be fine. Various kinds of resonators may cover various kinds of resonance frequencies of the seismic waves. There happens an energy dissipation of the seismic waves inside of the waveguide and the absorbed energy will turn into sound and heat. They make the temperature of the waveguide increasing depending on the magnitude of energy that arrives at the waveguide $[20,21]$.

A vertical view of the metamaterial barrier with many meta-cylinders are in Figure 3. The width of the barrier, $\Delta X$, is predicted in Equation (11). The depth of the waveguide should be at least the foundation work of the building to protect as in Figure 3, but it is not necessary to be more than the wavelength of the surface waves. The completed form of the waveguide is the aseismic cylindrical shell of many concentric rings in Figure 4.

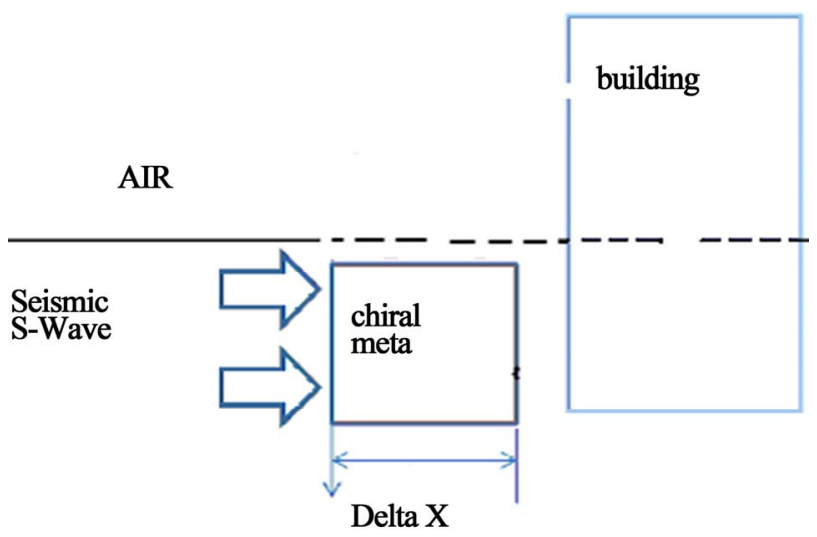

Figure 3. A vertical diagram of the chiral metamaterial (MTM) barrier and the building to protect from the seismic wave.

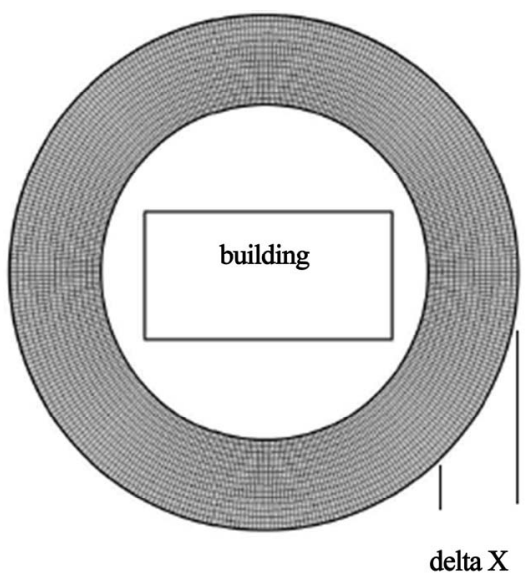

Figure 4. A sky view of a completed seismic barriers with many meta-cylinders.

Seismic waves cannot pass through water. Then, we can imagine a water barrier of a big trench filled with water. However, it is not stable to stand a series of attacks of "foreshock $\rightarrow$ main shock $\rightarrow$ aftershock." Because, if the outer part of the water trench is brought down by a seismic wave, we do not have enough time to rebuild it to prepare for the main shock and the after shock. Water cannot have a high refractive index $n$ in Equation (11), too. Maintaining the depth of the water trench up to the wavelength of the seismic wave require huge amount of water and, therefore, not be practical.

\section{Summary}

We introduced a supportive method for aseismic design. with an earthquake proof barrier around the building to be protected. This barrier is a kind of waveguide that reduces exponentially the amplitude of the dangerous seismic waves. Then, if the structures are protected with ground rubber layers would be possible to divert the $\mathrm{S}$ waves which do not impact a building.

The cloak can use giant-sized metamaterials that dampen the most destructive surface waves that are coupled to pressure and shear-body waves. Using large rings of a flexible metamaterials spaced around building foundations and tuned to the wavelength of the waves to be diverted, the researchers claimed the most destructive surface waves can be diverted around entire buildings.

Controlling the width, the chirality and refractive index of the waveguide, we can upgrade the aseismic range of the building as needed in order to defend it, at will, without touching it. It could be a big advantage of the waveguide method. This method will be effective for isolated buildings because we need some areas to construct the aseismic shell. It may be applicable for social overhead capitals such as power plants, dams, airports, nuclear reactors, oil refining complexes, long-span bridges, express rail-roads, etc. 


\section{REFERENCES}

[1] E. Majeswski, "Canonical Approach to Asymmetric Continua," In: R. Teisseyre, H. Nagahama and E. Majeswski, Eds., Physics of Asymmetric Continuum: Extreme and Fracture Processes-Earthquake Rotation and Soliton Waves, Springer, Berlin, 2008, pp. 209-218. doi:10.1007/978-3-540-68360-5_16

[2] V. Gioncu and F. M. Mazzolani, "Earthquake Engineering for Structural Design," Spon Press, New York, 2011, p. 223.

[3] R. Teisseyre, "New Developments in the Physics of Rotational Motions," Bulletin of the Seismological Society of America, Vol. 99, No. 2B, 2009, pp. 1028-1039.

[4] H. Torres-Silva and A. Souza de Assis, "Velocity and Gravitational Effects on GPS Satellites an Outline of Early Prediction and Detection of the Strong Earthquakes," Ingeniare, Vol. 18, No. 3, 2010, pp. 286-294.

[5] H. Torres-Silva, "Early Prediction and Detection of Strong Earthquakes through Chiral Radiation Waves," Journal of Vectorial Relativity, Vol. 6, No. 2, 2011, pp. 1-11.

[6] H. Torres-Silva and D. Torres Cabezas, "Chiral Changes on Ratio of the Compressional Velocity to the Shear Velocity of Earthquakes," International Journal of Research and Reviews in Applied Sciences, Vol. 12, No. 3, 2012, pp. 517-522.

[7] M. Farhat, S. Enoch, S. Guenneau and A. B. Movchan, "Broadband Cylindrical Acoustic Cloak for Linear Surface Waves in a Fluid," Physical Review Letters, Vol. 101, No. 13, 2008, Article ID: 134501. doi:10.1103/PhysRevLett.101.134501

[8] M. Farhat, S. Guenneau, S. Enoch and A. B. Movchan, "Cloaking Bending Waves Propagating in Thin Elastic Plates," Physical Review B, Vol. 79, No. 3, 2009, Article ID: 033102. doi:10.1103/PhysRevB.79.033102

[9] M. Farhat, S. Guenneau and S. Enoch, "Ultrabroadband Elastic Cloaking in Thin Plates," Physical Review Letters, Vol. 103, No. 2, 2009, Article ID: 024301. doi:10.1103/PhysRevLett.103.024301

[10] J. B. Pendry, "Negative Refraction Makes a Perfect Lens," Physical Review Letters, Vol. 85, No. 18, 2000, pp. 39663969. doi:10.1103/PhysRevLett.85.3966

[11] J. Zhu and G. V. Eleftheriades, "A Simple Approach for Reducing Mutual Coupling in Two Transmission-Line Lens," Physical Review Letters, Vol. 101, No. 1, 2008, Article ID: 013902. doi:10.1103/PhysRevLett.101.013902

[12] D. Schurig, et al., "Metamaterial Electromagnetic Cloak at Microwave Frequencies," Science, Vol. 314, No. 5801, 2006, pp. 977-980. doi:10.1126/science. 1133628

[13] H. Chen, B.-I. Wu and B. Zhang, "Electromagnetic Wave
Interactions with a Metamaterial Cloak," Physical Review Letters, Vol. 99, No. 6, 2007, Article ID: 063903. doi:10.1103/PhysRevLett.99.063903

[14] A. Demetriadou and J. B. Pendry, "Extreme Chirality in Swiss Roll Metamaterials," Physica B: Condensed Matter, Vol. 405, No. 14, 2009, pp. 2943-2946. doi:10.1016/j.physb.2010.01.009

[15] S. H. Kim and M. P. Das, "Seismic Waveguide of Metamaterial," Modern Physics Letters B, Vol. 26, No. 17, 2012, Article ID: 1250105.

[16] Y. Wu, Y. Lai and Z.-Q. Zhang, "Effective Medium Theory for Elastic Metamaterials in Two Dimensions," Physical Review B, Vol. 76, No. 20, 2007, Article ID: 205313. doi:10.1103/PhysRevB.76.205313

[17] Y. Wu, Y. Lai and Z.-Q. Zhang, "Elastic Metamaterials with Simultaneously Negative Effective Shear Modulus and Mass Density," Physical Review Letters, Vol. 107, No. 10, 2011, Article ID: 105506.

[18] X. Zhou and Z. Hu, "Analytic Model of Elastic Spheres," Physical Review B, Vol. 79, No. 19, 2009, Article ID: 195109. doi:10.1103/PhysRevB.79.195109

[19] J. B. Pendry, A. J. Holden, D. J. Robbins and W. J. Stewart, "Magnetism from Conductors and Enhanced Nonlinear Phenomena," IEEE Transactions on Microwave Theory and Techniques, Vol. 47, No. 11, 1999, pp. 20752084. doi: $10.1109 / 22.798002$

[20] C. Caloz and T. Itoch, "Electromagnetic Metamaterials," Wiley, New York, 2006.

[21] C. Ding, L. Hao and X. Zhao, "Two-Dimensional Acoustic Metamaterial with Negative Modulus," Journal of Applied Physics, Vol. 108, No. 7, 2010, Article ID: 074911. doi:10.1063/1.3493155

[22] L. L. Beranek, “Acoustics,” McGraw-Hill, New York, 1954.

[23] H. Torres-Silva, et al., "Chiral Waves in a Metamaterial Medium," International Journal of Pure and Applied Sciences and Technology, Vol. 2, No. 2, 2011, pp. 54-65.

[24] E. Plum, J. Zhou, J. Dong, V. A. Fedotov, T. Koschny, C. M. Soukolis and N. I. Zheledev, "Metamaterial with Negative Index Due to Chirality," Physical Review B, Vol. 79, No. 3, 2009, Article ID: 035407.

[25] J. Zhou, J. Dong, B. Wang, T. Koschny, M. Kafesaki and C. Soukoulis, "Negative Refractive Index Due to Chiraity," Physical Review B, Vol. 79, No. 12, 2009, Article ID: 121104. doi:10.1103/PhysRevB.79.121104

[26] H. Torres-Silva, "Chiral Transverse Electromagnetic Standing Waves with E II H in the Dirac Equation and the Spectra of the Hydrogen Atom," In: A. Akdagli, Ed., Behavior of Electromagnetic Waves in Different Media and Structures, Book Intech, Croatia, 2011, pp. 301-324. 